\title{
NONCONVEX BAYESIAN RESTORATION OF BLURRED FOREGROUND IMAGES
}

\author{
Chaoqun Dong* \\ Fraunhofer Singapore, Singapore
}

\begin{abstract}
Recently, some techniques for deblurring images having arbitrarily shaped boundaries have been proposed. However, when we observe a blurry foreground (FG) object on a sharp background (BG) there is no abrupt transition between them. Because previous techniques are not designed to cop with a smooth transition area between FG and BG, they avoid it by artificially discarding it from the computations. Here we construct, instead, an observation model that accounts for the occlusion photometric effect of the FG object on the $\mathrm{BG}$, and we illustrate its realism by comparing a simulation with a real capture. Then we use that observation model to pose a Bayesian MAP estimation problem with an L2-relaxed L0 sparse prior and two Gaussian likelihood terms (for the noise, and for the BG interference in the smooth transition area), which we solve by alternating optimizations. Using simulations, we demonstrate a higher performance of our method, compared to two state-of-the-art unconstrained boundary restoration techniques.
\end{abstract}

Index Terms - soft boundary restoration, blurred foreground image, non-convex optimization, Gaussian continuation

\section{INTRODUCTION}

In recent years we have witnessed significant advances in image restoration subject to different boundary conditions (BC) $[1,2,3,4$, $5,6,7,8,9]$. Besides overcoming the classical circular BC problem caused when using the Discrete Fourier Transform (DFT) to deconvolve images, these advances have also been applied to arbitrarily shaped supports, provided that boundaries were abrupt. The latter condition leaves out many real situations when an object in the foreground (FG) appears blurred (typically because of being out of focus and/or moving with respect to the visual field of the camera) on a sharp (on focus and static) background (BG). Although one can artificially create a "sharp boundary" for the pure FG pixels, by discarding the pixels mixing FG and BG (as done, e.g., in [4]), one would expect such information loss to damage the estimate quality. Here we explain, instead, a simplified optical model that describes how the BG and FG image components interact across the FG-BG transition area. We compare a real and a simulated observation, showing how the proposed observation model fits a real capture. In addition, by assuming Gaussian likelihood in the observation model both for the noise and for the BG interference on the FG, plus adding a previously proposed L2-relaxed sparsity-based prior [10, 11], we obtain a Maximum A Posteriori (MAP) cost function. We (locally, in principle) minimize that function by splitting variables and alternating marginal optimizations. Our results in color simulations surpass current (sharp-boundary-based) state-of-the-art of [4] and [7].

\footnotetext{
*Supported by the National Research Foundation, Prime Minister's Office, Singapore, under International Research Centres in Singapore Funding Initiative.

${ }^{\dagger}$ Funded by the Spanish Government grant FIS2016-75891-P.
}

\author{
Sergio Barbero, Javier Portilla ${ }^{\dagger}$ \\ Instituto de Óptica, CSIC, Madrid
}

\section{OBSERVATION MODEL}

Our goal in this section is to model how the (blurred) FG and the (sharp) BG image components interact across their transition area. Given a sharp BG image $f_{B G}(x, y)$ (on focus, static, with no occluding FG), and a sharp FG image $f_{F G}(x, y)$ (again, on focus) on a black BG, we model the observation $g(x, y)$ portraying an out-offoucs $\mathrm{FG}^{1}$ on the sharp BG. Here we consider a (rotationally symmetric) paraxial geometrical optical model, i.e. free from aberrations and diffraction.

First, the out-of-focus FG object can be modeled, in a first (classical) approximation, for not very wide angles, as the convolution of an assumed spatially invariant Point Spread Function (PSF) $h(x, y)$ (the blurring kernel at that distance) with $f_{F G}(x, y)$. Because of the blur, the observed boundaries of the FG silhouette are not abrupt, but smooth. In addition, they are also semi-transparent, i.e., across those boundaries, $f_{B G}(x, y)$ gets mixed with the blurred FG. According to the model assumptions, the intensities from FG and BG are simply added. The light coming from the BG is partially occluded by the FG object, and, thus, $f_{B G}(x, y)$ is attenuated by a certain occlusion factor $m_{o}(x, y)$, so we can write (see, e.g., $\left.[12,13,14,15]\right)$ :

$$
g(x, y)=f_{F G}(x, y) \star h(x, y)+m_{o}(x, y) f_{B G}(x, y)+w(x, y),
$$

where $w(x, y)$ refers to sensor noise, which we will model as zeromean Gaussian of known variance $\sigma_{w}^{2}$. We also assume the PSF $h(x, y)$ has been previously estimated (see optical model in next section). Our last assumption is that we know, or can estimate, the silhouette of $f_{F G}(x, y)$ from the observation (defocus matting problem, see e.g. [13, 14, 15]), which we term $m(x, y)$.

\subsection{Calculating the FG occlusion effect on the BG}

To determine $m_{o}(x, y)$, the occlusion mask of Eq. (1), we build a simple optical model comprising the following known values: entrance pupil radius $(E)$ and focal length $(f)$ of the camera, plus the distances $d_{B G}, d_{F G}$ of the BG and FG planes, respectively, to the entrance pupil. Figure 1 represents an optical system focusing on the BG plane, including an out-of-focus, FG object, closer to the camera. Considering similar triangles and paraxial imaging relations [16], all involved quantities can be computed from $E, f, d_{B G}$ and $d_{F G}$, including the defocus kernel radius (denoted by $h$ in the figure). From now on we will denote $\left(x_{o}, y_{o}\right),\left(x_{p}, y_{p}\right)$ and $(x, y)$ the points on the BG, pupil and image planes, respectively. The proportion of light emitted from a point $\left(x_{o}, y_{o}\right)$ on the BG plane and occluded by the FG is:

$$
m_{o}^{\prime}\left(x_{o}, y_{o}\right)=\frac{\iint E\left(x_{p}, y_{p}\right) m_{x_{o}, y_{o}}\left(x_{p}, y_{p}\right) d x_{p} d y_{p}}{\iint E\left(x_{p}, y_{p}\right) d x d y},
$$

\footnotetext{
${ }^{1} \mathrm{~A}$ totally parallel discussion applies to the motion blur case.
} 


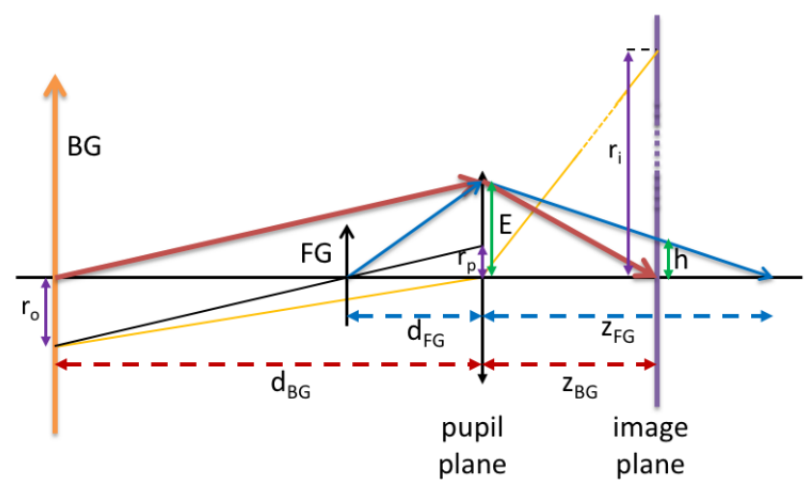

Fig. 1. Optics scheme (on-focus BG, out-of-focus FG) relating axial distances and radial coordinates.

where, by a slight abuse of previous notation, $E\left(x_{p}, y_{p}\right)$ denotes here a binary pupil function, and $m_{x_{o}, y_{o}}\left(x_{p}, y_{p}\right)$ represents the (also binary) FG silhouette projected from $\left(x_{o}, y_{o}\right)$ onto the entrance pupil. Considering $d_{B G} / d_{F G}>>1$, for moderate fields of view, the change in shape of $m_{x_{o}, y_{o}}\left(x_{p}, y_{p}\right)$ with angle of incidence is small, whereas its relative position may change a lot. Therefore, if we call $m_{0,0}\left(x_{p}, y_{p}\right)$ the silhouette of the FG object under normal incidence, we can approximate $m_{x_{o}, y_{o}}\left(x_{p}, y_{p}\right) \simeq m_{0,0}\left(x_{p}-k_{o p} x_{o}, y_{p}-k_{o p} y_{o}\right)$ (where $k_{o p}=r_{p} / r_{o}=\left(d_{B G} / d_{F G}-1\right)^{-1}$, see Fig. 1$)$, and previous expression becomes a convolution:

$$
m_{o}^{\prime}\left(x_{o}, y_{o}\right) \simeq m_{0,0}\left(k_{o p} x_{o}, k_{o p} y_{o}\right) \star \frac{E\left(k_{o p} x_{o}, k_{o p} y_{o}\right)}{\iint E\left(x_{p}, y_{p}\right) d x_{p} d y_{p}} .
$$

To translate this equation from BG object coordinates into image coordinates, we apply paraxial optics relations and similar triangles, to obtain $k_{i o}=r_{o} / r_{i}=d_{B G} / f-1$, and $h=k_{i p}^{-1} E$ (scalar values, see Fig. 1), being $k_{i p}=k_{i o} k_{o p}$. Furthermore, provided the optical system is rotationally symmetric, one can generalize the previous $E / h$ scaling to a functional scaling for arbitrary pupil functions: $h(x, y) \propto E\left(k_{i p} x, k_{i p} y\right)$. By using these relations in Eq. (3) we obtain the occlusion mask in the image, $m_{o}(x, y)=m_{o}^{\prime}\left(k_{i o} x, k_{i o} y\right)$ :

$$
m_{o}(x, y) \simeq m(x, y) \star h(x, y),
$$

with $m(x, y)=m_{0,0}\left(k_{i p} x, k_{i p} y\right)$ being the binary (sharp) FG mask as it would be seen on the image plane ${ }^{2}$. Finally, by using Eqs.(4) and (1), we obtain the complete image formation model:

$$
\begin{aligned}
g(x, y)= & f_{F G}(x, y) \star h(x, y)+ \\
& (m(x, y) \star h(x, y)) f_{B G}(x, y)+w(x, y) .
\end{aligned}
$$

Although this result is well-known (see $[12,14,15,9]$ ) (and applicable as well to moving FG $[12,9])$, we have not found its explicit and complete optical derivation (some partial analysis can be found in $[17,13])$. We illustrate this observation model in Fig. $2^{3}$. By comparing the simulation (g) (here without added noise) with its real observation counterpart (h) we can appreciate the realism of the model.

\footnotetext{
${ }^{2}$ Note that the integral of the denominator in Eq. (3) is absorbed into $h(x, y)$, since $h(x, y)$, by convention, integrates to one.

${ }^{3}$ We captured (a), and (b)+(h) using $f=50 \mathrm{~mm}, f / 3.5, d_{F G} \approx 2.00 \mathrm{~m}$, $d_{B G} \approx 12.33 \mathrm{~m}$, shutter $t=1 / 160 \mathrm{~s}$, with Canon EOS 1D Mark III, pixel pitch $7.2 \mu \mathrm{m}$. All calculations have been done in the linearized color space. Sharp FG image (Fig. 2(a)) has a relative magnification of $z_{F G} / z_{B G} \approx$ $1 / 0.98$, which has been numerically corrected $(\times 0.98)$.
}

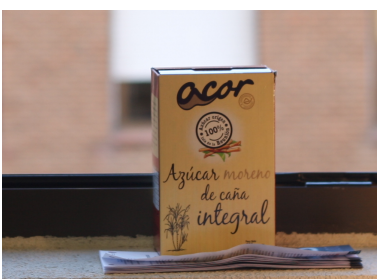

(a)

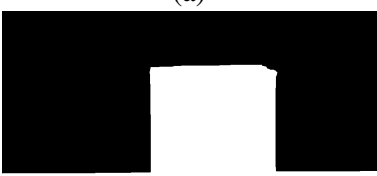

(c)

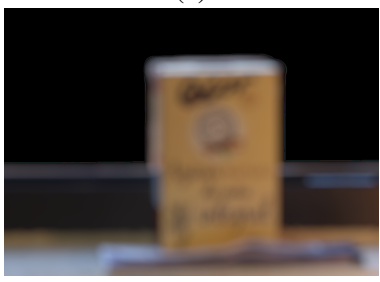

(e)

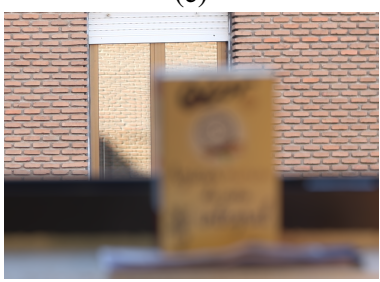

$(\mathrm{g})$

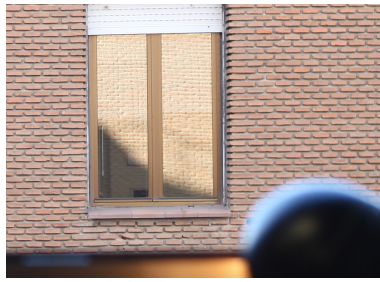

(b)

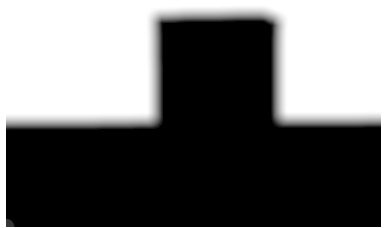

(d)

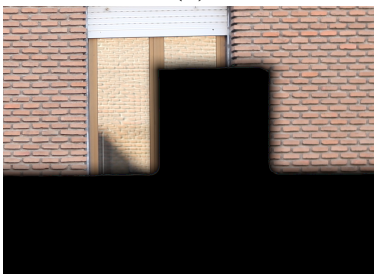

(f)

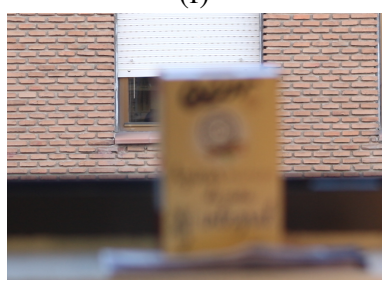

(h)
Fig. 2. (a) Real image, focus on FG; (b): real image, focus on BG; (c): segmentation FG/BG mask from (a); (d): $m_{o}$ is (1-(c)) convolved with $h$; (e): result of convolving $((\mathrm{a}) \times(\mathrm{c}))$ with $h$; (f): (b) $\times(\mathrm{d})$; (g): simulation result: (e) $+(\mathrm{f})$; (h): real image, focus on BG ((b) \& (h) are slightly overlapping crops from same picture).

\section{MAXIMUM A POSTERIORI ESTIMATION}

Terming $b_{F G}$ the blurred and noisy observed FG object, from Eq. (5), we have $b_{F G}=f_{F G} \star h+w,{ }^{4}$ which in matrix and vector notation becomes

$$
\mathbf{b}=\mathbf{H} \mathbf{f}+\mathbf{w}
$$

where $\mathbf{f}$ is a vectorized form of $f_{F G}$. Then, again from the observation model (Eq. (5)) we have:

$$
\mathbf{g}=\mathbf{b}+\mathbf{D}_{m_{o}} \mathbf{c},
$$

where $\mathbf{c}$ is a vectorized notation for $f_{B G}$, and $\mathbf{D}_{m_{o}}$ is a diagonal matrix reproducing the soft occlusion effect on the background (Eq. (4)). Our goal in this paper is to estimate both $\mathbf{f}$ and $\mathbf{c}$ (the latter unknown only in the transition area), given the observation $\mathbf{g}$, and assuming we know $m(x, y), h(x, y)$ and $\sigma_{w}^{2}$.

\footnotetext{
${ }^{4}$ For convenience we have assigned here the noise term $\mathbf{w}$ to the FG support area, thus ignoring the (here irrelevant) effect of the noise on the BG.
} 


\subsection{Problem formulation}

Our main calculation is obtaining a maximum a posteriori (MAP) estimate of the sharp FG, $\hat{\mathbf{f}}$, by performing a simultaneous optimization on $\mathbf{f}$ and $\mathbf{b}$. We achieve that by injecting prior information from an L2-relaxed analysis-based sparse image model of our own [10,11] into the previously described observation model. Having $\hat{\mathbf{f}}$ we estimate the BG in the transition area, $\hat{\mathbf{c}}$, by using Eq. (6) and Eq. (7):

$$
\hat{\mathbf{c}}=\mathbf{D}_{m_{o}}^{\#}(\mathbf{g}-\hat{\mathbf{b}}),
$$

where $\mathbf{D}_{m_{o}}^{\#}$ is the pseudo-inverse of $\mathbf{D}_{m_{o}}$, and $\hat{\mathbf{b}}=\mathbf{H} \hat{\mathbf{f}}$ provides us with an estimate of $\mathbf{b}$ in the transition area.

For the MAP estimation we use:

$$
\begin{aligned}
(\hat{\mathbf{f}}, \hat{\mathbf{b}}) & =\arg \max _{\mathbf{f}, \mathbf{b}} p(\mathbf{f}, \mathbf{b} \mid \mathbf{g}) \\
& =\arg \max _{\mathbf{f}, \mathbf{b}} p(\mathbf{f}, \mathbf{b}, \mathbf{g}) \\
& =\arg \max _{\mathbf{f}, \mathbf{b}} p(\mathbf{g} \mid \mathbf{f}, \mathbf{b}) p(\mathbf{f}, \mathbf{b}) \\
& =\arg \max _{\mathbf{f}, \mathbf{b}} p(\mathbf{g} \mid \mathbf{b}) p(\mathbf{b} \mid \mathbf{f}) p(\mathbf{f}),
\end{aligned}
$$

where we have applied that $\mathbf{g}$ is conditionally independent on $\mathbf{f}$ given b (see Eq. (7)). We used simple Gaussian likelihood terms for the noise in Eq. (6) and for the "BG interference" in Eq. (7), obtaining:

$$
\begin{gathered}
-\log p(\mathbf{b} \mid \mathbf{f})=\frac{1}{2 \sigma_{w}^{2}}\|\mathbf{b}-\mathbf{H} \mathbf{f}\|^{2}+C_{1} \\
-\log p(\mathbf{g} \mid \mathbf{b})=\frac{1}{2 \sigma_{B G}^{2}}\left\|\mathbf{D}_{m_{o}}^{-1}\left(\mathbf{b}-\left(\mathbf{g}-\mathbf{D}_{m_{o}} \mu_{B G}\right)\right)\right\|^{2}+C_{2},
\end{gathered}
$$

where $\mu_{B G}, \sigma_{B G}^{2}$ are measurements of the mean and variance on the BG. For the prior $p(\mathbf{f})$ we used the formulation from [11]:

$$
-\log p(\mathbf{f})=\min _{\mathbf{a}}\left\{\frac{1}{\alpha}\|\mathbf{a}\|_{0}+\frac{1}{2 \sigma_{r}^{2}}\left\|\mathbf{a}-\Phi^{*} \mathbf{f}\right\|^{2}\right\}+C_{3},
$$

where $\Phi^{*}$ is a linear sparsifying representation. ${ }^{5}$ Finally, we impose $\mathrm{f}$ to be within the non-zero support of the sharp FG mask $m(x, y)$ : $\mathbf{f}=\mathbf{D}_{m} \mathbf{f}$. Using the four previous equations we obtain:

$$
\begin{aligned}
(\hat{\mathbf{f}}, \hat{\mathbf{b}}, \hat{\mathbf{a}}) & =\arg \min _{\mathbf{f}, \mathbf{b}, \mathbf{a}} \frac{1}{2 \sigma_{B G}^{2}}\left\|\mathbf{D}_{m_{o}}^{-1}\left(\mathbf{b}-\left(\mathbf{g}-\mathbf{D}_{m_{o}} \mu_{B G}\right)\right)\right\|^{2} \\
& +\frac{1}{2 \sigma_{w}^{2}}\|\mathbf{b}-\mathbf{H f}\|^{2}+\frac{1}{\alpha}\|\mathbf{a}\|_{0} \\
& +\frac{1}{2 \sigma_{r}^{2}}\left\|\mathbf{a}-\Phi^{*} \mathbf{f}\right\|^{2}, \text { s.t. } \mathbf{f}=\mathbf{D}_{m} \mathbf{f}
\end{aligned}
$$

\subsection{Algorithm}

To attack Eq. (13) we do alternating marginal optimizations of the involved variables [18]. By differentiation w.r.t. $\mathbf{b}, \mathbf{a}$ and $\mathbf{f}$ in Eq. (13), equating to zero and solving, respectively, plus imposing that $\mathbf{f}$ lies within the $m(x, y)=1$ support, we obtain the algorithm (Eqs. (14)). First step is a recalculation of the blurred FG $\mathbf{b}^{(k)}$ via a re-weighted sum of the observed coefficients $\mathbf{g}$ (after subtracting BG's mean) and the blurred current estimate of the FG, $\mathbf{H} \mathbf{f}^{(k)}$. Second and third steps are the same as in [11]: a thresholding using $T=\sqrt{2 \alpha / \sigma_{r}^{2}}$ on the analysis coefficients, and a linear adjustment that can be efficiently

\footnotetext{
${ }^{5}$ In fact, we used three combined linear representations, same as in [11], which we have summarized here to a single one, for notation simplicity.
}

performed in the Fourier domain. Such adjustment corresponds to the optimal trade-off between the likelihood term (Eq. (10)) and the sparse prior (Eq. (12)). Fourth step imposes the sharp FG spatial support $m$ to current FG estimation $\mathbf{f}^{(k+1)}$.

$$
\begin{aligned}
& \mathbf{b}^{(k)} \leftarrow\left(\frac{1}{\sigma_{B G}^{2}} \mathbf{D}_{m_{o}}^{-2}+\frac{1}{\sigma_{w}^{2}} \mathbf{I}\right)^{-1} \times \\
&\left(\frac{1}{\sigma_{B G}^{2}} \mathbf{D}_{m_{o}}^{-2}\left(\mathbf{g}-\mathbf{D}_{m_{o}} \mu_{B G}\right)+\frac{1}{\sigma_{w}^{2}} \mathbf{H} \mathbf{f}^{(k)}\right) \\
& \mathbf{a}^{(k)} \leftarrow \Theta_{H}\left(\Phi^{*} \mathbf{f}^{(k)}, T\right)\left(\frac{1}{\sigma_{w}^{2}} \mathbf{H}^{*} \mathbf{H}+\frac{1}{\sigma_{r}^{2}} \Phi \Phi^{*}\right)^{-1} \times \\
& \mathbf{f}^{(k+1)} \leftarrow\left(\frac{1}{\sigma_{w}^{2}} \mathbf{H}^{*} \mathbf{b}^{(k)}+\frac{1}{\sigma_{r}^{2}} \Phi \mathbf{a}^{(k)}\right) \\
& \mathbf{f}^{(k+1)} \leftarrow \mathbf{D}_{m} \mathbf{f}^{(k+1)}, k \leftarrow k+1 .
\end{aligned}
$$

\subsubsection{Local vs. Global Optimization}

Previous updating rules are only guaranteed to converge to a local minimum of the cost function (Eq.(13)). Thus, not just convergence speed, but the optimization result itself benefits from having a good initial guess. We have used our Spectral Pre-Adaptation (SPA) technique $[5,6,7]$ for a first estimate $\mathbf{b}^{(0)}$ of $\mathbf{b}$. SPA extends a given blurred observation with arbitrary boundaries, for a known blurred kernel, to an image with circular BC on a rectangular support. Because it can not handle "soft boundaries", we must take, for the image being extended, the inner part of the FG corresponding to $m_{o}=1$. We have termed this method "LSBR", for Local Soft Boundary Restoration. However, when the initial guess $\mathbf{b}^{(0)}$ is poor the LSBR solution can be far from the global optimum. A classical strategy to avoid getting trapped into unfavorable local minima is to express the uncertainty about the current estimate as an increased value of the noise variance w.r.t. to its nominal value (sometimes termed graduated non-convexity or homotopy continuation, being also connected to deterministic annealing, see e.g. $[19,20,21,22])$. In particular, here we have used the exponential decaying rule $\tilde{\sigma}_{w}^{2}(n)=\left(K \rho^{n}+1\right) \sigma_{w}^{2}$, and substituted $\tilde{\sigma}_{w}^{2}$ by $\sigma_{w}^{2}$ in the algorithm (Eqs.(14)). We have termed this method's variant Global Soft Boundary Restoration (“GSBR").

\section{EXPERIMENTS, RESULTS AND DISCUSSION}

We carried out three restorations on simulated color observations ${ }^{6}$ for comparing the performance of LSBR and GSBR to two methods used for restoring arbitrary-shaped images, but not designed to cop with soft boundaries, namely: (1) the Spectral Pre-Adaptation method $[5,6,7]^{7}$ combined with deblurring (using [11]), and (2) the UBC-ADMM method [4]. All methods have been implemented in the same way described in section 3.1: first the FG (its blurred version, in the SPA case) is estimated and then, based on the blurred FG estimate, the BG is estimated (Eq. (8)). Note that, for the FG estimation, both SPA and UBC-ADMM only use the FG pixels with total occlusion $\left(m_{o}=1\right)$, whereas our method uses all pixels with some occlusion $\left(m_{o}>0\right)$. For all three experiments we have simulated the defocus kernel using circular disks, and have considered

\footnotetext{
${ }^{6}$ Image origins. (1) FG: SIGN: "no-fishing-sign", www. publicdomainpictures. net; THUMB UP: "good job", bought by J. Portilla in www. istockphoto. com; BOX: J. Portilla. (2) BG: J. Portilla \& C. Dong.

${ }^{7}$ We are thankful to F. Sroubek for adapting our SPA code to use conjugate gradients instead of our previous, less robust, optimization method.
} 


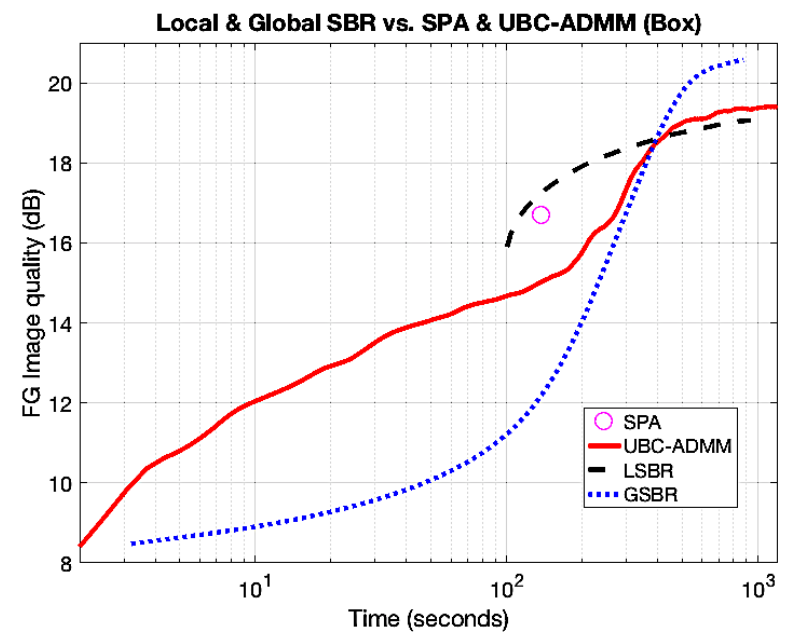

Fig. 3. Numerical performance (FG SNR vs. time) for $B O X$.

\begin{tabular}{|l|c|c|c|c|}
\hline & SPA & ADMM & LSBR & GSBR \\
\hline SIGN & $23.1-36.8$ & $24.2-46.1$ & $\mathbf{2 4 . 3}-38.6$ & $24.2-38.6$ \\
THUMB UP & $26.8-37.1$ & $27.7-38.3$ & $\mathbf{2 8 . 4 - 4 3 . 6}$ & $\mathbf{2 8 . 4 - 4 2 . 7}$ \\
BOX & $16.7-32.4$ & $19.6-33.3$ & $18.9-34.5$ & $\mathbf{2 0 . 7 - 3 5 . 9}$ \\
\hline
\end{tabular}

Table 1. Signal-to-noise ratios (FG-BG in $\mathrm{dB}$ ) of compared results.

only quantization noise (either 8 or 16 bits). First experiment (SIGN image) involves a simple FG image with a convex shape (a perfect rectangle), and a moderate amount of blur (diameter $D=13$ pixels). Second experiment (THUMB UP image) depicts an FG object with a non-convex shape, including local structures comparable in size to the (now larger) blurring kernel $(D=19)$. In this case and the next one, we have segmented the FG mask from a real image. Third experiment (BOX image) corresponds to the scene portrayed in Fig.1(g), for convenience in half its original resolution. We used a defocus diameter of $D=21$ pixels. Same as before, we have considered the $s R G B$ mapping (gamma correction), now both for the image simulation and the restoration. In the first two simulations we have simulated 8-bit quantization, whereas in the third one we used 16 bits. In the latter case, due to the non-linear gamma mapping, noise variance in the linear RGB space has needed to be estimated from the linearized observation by using the median absolute deviation (MAD) method [23]. We see, in Table 1 and Fig. 4, that SPA obtains results significantly worse than UBC-ADMM and the two variants of SBR. For the SIGN image, having the FG a moderate amount of blur, a simple support and cartoon-like content, the proposed methods do not significantly improve on the excellent UBC-ADMM result. For THUMB UP, though, with more blur and more complex FG (shape and content), SBR methods behave better than UBC-ADMM. Comparing Fig. 4(f) and (g) we see how LSBR, thanks to its texture-friendly L2-relaxed L0 prior, does not produce the typical TV false contour artifacts, visible in UBC-ADMM. Finally, for the more difficult experiment $B O X$, we see how SPA fails to provide a good enough initial guess for LSBR. Then, it is necessary to use the global minimization strategy of GSBR to arrive to a more favorable result (compare Fig. 4(k) and (l)). In Fig. 3 we plot the performance (FG SNR vs. time) of the compared methods. ${ }^{8}$

${ }^{8} \mathrm{SPA}$ result is plotted as a single dot because it estimates $\mathbf{b}$ which is deconvolved at convergence. Abscissa axis is time and not \#iterations, because UBC-ADMM's iterations do not have the same complexity as SBR's.

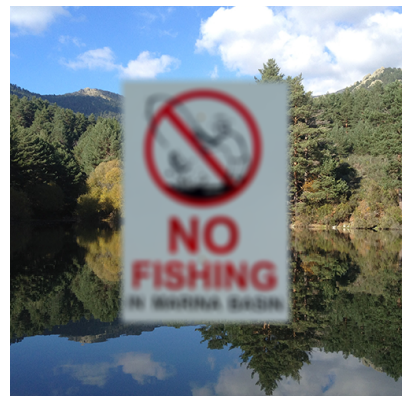

(a) Simulated observation

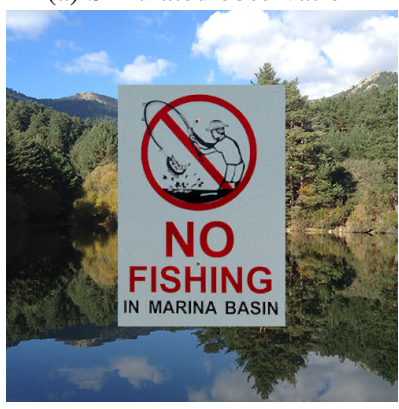

(c) UBC-ADMM result nage restoration problems. Our tormulation of a $\ell_{2}$-rela
0 pseudo-norm prio allows for an especially simple maxim
posteriori estimation rative marginal optimization algorit posteriori estimation ative marginal optimization algor:

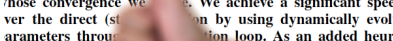
arameters throu
wist, we fix in
mpirically optim mpirically optimi
erformance benc

ethod is not just fast and nd flexible. First, it is able to prowe.

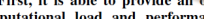

(e) Simulated observation nage restoraton preplems. Our tormulaton or a $\ell_{2}$-ree
0 pseudo-norm prio tllows for an especially simple maxi posteriori estimation "utive marginal optimization algorit those convergence wo We achieve a significant spee wist, we fix in wist, we fix ix
mpirically opt
s. erformance benc telod is not just tast and effere is able to provide an

(g) LSBR result

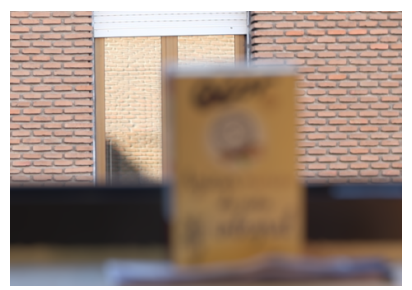

(i) Simulated observation

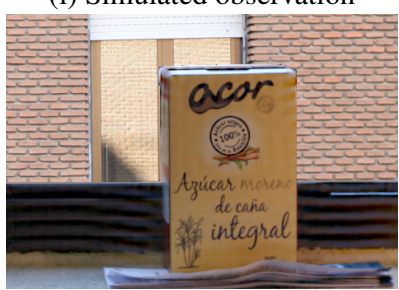

(k) LSBR result ver the direct is a by using dynamically ever

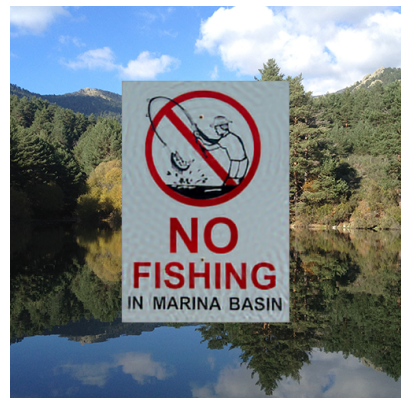

(b) SPA result

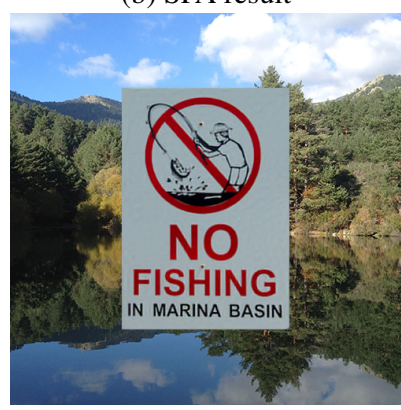

(d) LSBR result

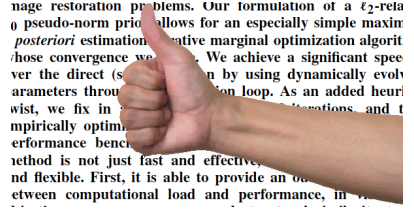

(f) UBC-ADMM result nage restoration problems. Uur tormulaton of a $\varepsilon_{2}$-rel

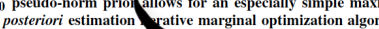
hose convergence we
ver the direct (st we achieve a significant spee ver the direct (st
arameters throug wist, we fix in at mpirically optimi erformance bench iethod is not just fast and effective, etween computational load and performoumen

(h) Background Estimate

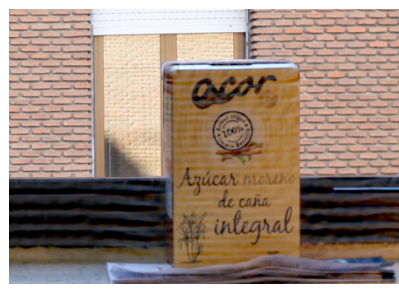

(j) SPA result

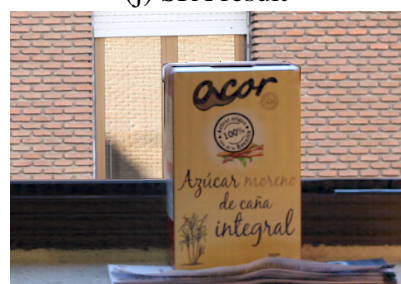

(l) GSBR result
Fig. 4. Visual results for $S I G N, T H U M B U P$ and $B O X$ compositions. 


\section{REFERENCES}

[1] S. J. Reeves, "Fast image restoration without boundary artifacts," IEEE Transactions on Image Processing, vol. 14, no. 10, pp. 1448-1453, Oct 2005.

[2] M. Sorel, "Removing boundary artifacts for real-time iterated shrinkage deconvolution," IEEE Transactions on Image Processing, vol. 21, no. 4, pp. 2329-2334, April 2012.

[3] A. Matakos, S. Ramani, and J. A. Fessler, "Accelerated edge-preserving image restoration without boundary artifacts," IEEE Transactions on Image Processing, vol. 22, no. 5, pp. 2019-2029, May 2013.

[4] M. S. C. Almeida and M. Figueiredo, "Deconvolving images with unknown boundaries using the alternating direction method of multipliers," IEEE Transactions on Image Processing, vol. 22, no. 8, pp. 3074-3086, Aug 2013.

[5] J. Portilla, "Maximum likelihood extension for non-circulant deconvolution," in Image Processing (ICIP), 2014 IEEE International Conference on, Oct 2014, pp. 4276-4279.

[6] C. Dong and J. Portilla, "Maximum likelihood interpolation for aliasing-aware image restoration," in 2016 IEEE International Conference on Image Processing (ICIP), Sept 2016, pp. 564568.

[7] C. Dong and J. Portilla, "Spectral pre-adaptation for twostep arbitrary-shape-support image restoration," in 2017 IEEE International Conference on Image Processing (ICIP), Sep. 2017, pp. 3515-3519.

[8] J. Kotera, V. Smidl, and F. Sroubek, "Blind deconvolution with model discrepancies," IEEE Transactions on Image Processing, vol. 26, no. 5, pp. 2533-2544, May 2017.

[9] J. Kotera and F. Sroubek, "Motion estimation and deblurring of fast moving objects," in 201825 th IEEE International Conference on Image Processing (ICIP), Oct 2018, pp. 2860-2864.

[10] J. Portilla, "Image restoration through L0 analysis-based sparse optimization in tight frames," in 2009 16th IEEE International Conference on Image Processing (ICIP), Nov 2009, pp. 3909-3912.

[11] J. Portilla, A. Tristan-Vega, and I.W. Selesnick, "Efficient and robust image restoration using multiple-feature L2-relaxed sparse analysis priors," Image Processing, IEEE Transactions on, vol. 24, no. 12, pp. 5046-5059, Dec 2015.

[12] S. K. Nayar and M. Ben-Ezra, "Motion-based motion deblurring," IEEE Transactions on Pattern Analysis and Machine Intelligence, vol. 26, no. 6, pp. 689-698, June 2004.

[13] M. McGuire, W. Matusik, H. Pfister, J. F. Hughes, and F. Durand, "Defocus video matting," ACM Trans. Graph., vol. 24, no. 3, pp. 567-576, July 2005.

[14] N. Joshi, W. Matusik, S. Avidan, H. Pfister, and W. T. Freeman, "Exploring defocus matting: Nonparametric acceleration, super-resolution, and off-center matting," IEEE Computer Graphics and Applications, vol. 27, no. 2, pp. 43-52, March 2007.

[15] N. Xu, B. L. Price, S. Cohen, and T. S. Huang, "Deep image matting," CoRR, vol. abs/1703.03872, 2017.

[16] V. N. Mahajan, Optical imaging and aberrations I. Ray geometrical optics, SPIE Optical Engineering Press, Bellingham, Washington, USA., 1998.
[17] N. Asada, H. Fujiwara, and T. Matsuyama, "Seeing behind the scene: Analysis of photometric properties of occluding edges by the reversed projection blurring model," IEEE Trans. Pattern Anal. Mach. Intell., vol. 20, no. 2, pp. 155-167, Feb. 1998.

[18] R. Glowinski, S.J. Osher, and W. Yin, Splitting Methods in Communication, Imaging, Science, and Engineering, Scientific Computation. Springer International Publishing, 2017.

[19] A. Blake and A. Zisserman, "Visual reconstruction," 1987.

[20] H. Mobahi and J.W. Fisher, "A theoretical analysis of optimization by Gaussian continuation," in $A A A I, 2015$.

[21] N. Ueda and R. Nakano, "Deterministic annealing EM algorithm," Neural Networks, vol. 11, no. 2, pp. 271 - 282, 1998.

[22] L. Mancera and J. Portilla, "Non-convex sparse optimization through deterministic annealing and applications," in 2008 15th IEEE International Conference on Image Processing, Oct 2008, pp. 917-920.

[23] Frank R. Hampel, "The influence curve and its role in robust estimation," Journal of the American Statistical Association, vol. 69, no. 346, pp. 383-393, 1974. 